\title{
Combining Cryptography with EEG Biometrics
}

\author{
Robertas Damaševičius (iD), Rytis Maskeliūnas $(\mathbb{D})^{2}$ \\ Egidijus Kazanavičius, ${ }^{2}$ and Marcin Woźniak ${ }^{3}$ \\ ${ }^{1}$ Department of Software Engineering, Kaunas University of Technology, Studentu 50-415, Kaunas, Lithuania
${ }^{2}$ Centre of Real Time Computer Systems, Kaunas University of Technology, K. Baršausko 59-A338, Kaunas, Lithuania
${ }^{3}$ Institute of Mathematics, Silesian University of Technology, Kaszubska 23, 44-100 Gliwice, Poland
}

Correspondence should be addressed to Robertas Damaševičius; robertas.damasevicius@ktu.lt

Received 22 December 2017; Revised 20 March 2018; Accepted 18 April 2018; Published 22 May 2018

Academic Editor: Ivan Volosyak

Copyright (C) 2018 Robertas Damaševičius et al. This is an open access article distributed under the Creative Commons Attribution License, which permits unrestricted use, distribution, and reproduction in any medium, provided the original work is properly cited.

Cryptographic frameworks depend on key sharing for ensuring security of data. While the keys in cryptographic frameworks must be correctly reproducible and not unequivocally connected to the identity of a user, in biometric frameworks this is different. Joining cryptography techniques with biometrics can solve these issues. We present a biometric authentication method based on the discrete logarithm problem and Bose-Chaudhuri-Hocquenghem $(\mathrm{BCH})$ codes, perform its security analysis, and demonstrate its security characteristics. We evaluate a biometric cryptosystem using our own dataset of electroencephalography (EEG) data collected from 42 subjects. The experimental results show that the described biometric user authentication system is effective, achieving an Equal Error Rate (ERR) of 0.024 .

\section{Introduction}

Brain computer interface (BCI) is a highly growing field of research with application in healthcare systems (from fall prevention to neuronal rehabilitation) to educational, selfregulation, production, marketing, and security as well as games and entertainment. BCI aims to provide a channel of communication that does not depend on the usual use of peripheral nerves and muscles [1]. While the main intended target application for BCI research is the development of motor function independent prosthetic devices for impaired patients, other applications of BCI, such as those for learning [2], gaming [3, 4], or entertainment [5], raise the need for ensuring the security and privacy of subjects using BCI systems. BCI systems are based on measurement of brain activity on the surface (in case of noninvasive $\mathrm{BCI}$ ) or inside (in case of invasive $\mathrm{BCI}$ ) of the human skull using electrodes. The results of the measurement represent the sum of electrical impulses emitted by a large number of brain's neurons. Noninvasive EEG signal is recorded by attaching the electrodes to the head of a subject according to a given map such as the 10-20 international system for the placement of EEG electrodes.
Recently, BCI applications for biometrics have attracted increased attention from the researchers. Biometrics provides means for identifying people based on their physiological characteristics [6]. Recently, there has been tremendous growth in research on cryptography and biometric frameworks because of incredible need for data security in numerous applications, such as e-commerce, e-health, egovernment, e-voting, blockchain, law enforcement, digital forensics, and homeland security. The goal is to verify the identity of a subject using some characteristic of a subject. In cryptographic frameworks, users use their passwords or secret keys to protect their confidential data. However, the use of passwords for identification has some wellknown drawbacks: textual passwords can be spied over or cracked, and secret keys are too long and difficult to memorize and can be stolen if stored somewhere. The downside of cryptography is that verification strategies are not unequivocally connected to the person identity. Unlike cryptography based authentication methods, biometrics using behavioural and physiological characteristics such as iris, fingerprints, electroencephalography (EEG) data, face, palm, voice, and gait, is convenient and cannot be forgotten or lost. 
The EEG-based subject identification is relatively new. The advantages of using EEG for biometrics are its low exposability (cannot be casually obtained or stolen by external observers) and resistance to forced extraction because understress brain activity changes [7]. They also can be used by disabled patients or users missing some physical trait. Efforts to develop biometric methods and systems based on the EEG have targeted the development of subject condition monitoring tools, for example, for detection of sleep apnea [8], schizophrenia [9], or epilepsy [10]; the creation of BCI systems to assist disabled people [11]; and marketing [12]. Analysts predict that the global EEG and electrocardiography (ECG) biometrics market is to expand at a compound annual growth rate of $12.37 \%$ during the period $2016-2020$ [13].

The suitability of using EEG for privacy and security applications can be attributed to morphological, anatomical, and functional plasticity (behaviour-related lasting changes in functional connections) traits [14], which contribute to discriminability between subjects [15]. Several studies (mainly from the fields of human physiology and genetics) have confirmed that the spectral characteristics of the EEG alpha waves (in the $8-12 \mathrm{~Hz}$ range, which reflect relaxation and disengagement) and the beta waves (in the $12-30 \mathrm{~Hz}$ range, related to action and concentration) of EEG show the strongest heritability relationship [16].

The difficulties related to using EEG data are its instability over time (the EEG permanence problem [17]). It is still difficult to achieve high accuracy of EEG-based biometric systems, which motivate researchers to explore new EEG data analysis methods. However, the research community still lacks knowledge on specific discriminant features of EEG suable for biometry [18]. Up to now, the EEG power spectrum features were used to achieve relatively good classification performance [18]. Several methods, which focus on the concepts and methods adopted from the network science, such as functional connectivity [19] and network organization [20], have been proposed. Fuzzy commitment (FC) scheme [21] can be used as a theoretical background for combining cryptography and biometrics. In the FC scheme, a secret key is linked to the reference biometric template, and the difference vector is calculated in such way that the secret key may be restored using the difference vector and the query biometric template. Another approach is a fuzzy vault (FV) based on polynomial reconstruction [22]. The FC and FV schemes have been applied to biometrics before $[23,24]$.

Here we propose a secure EEG-based cryptographic authentication scheme based on the commitment scheme adopted from [25], provide a theoretical analysis of the security characteristics of the proposed scheme, apply the scheme to biometric systems to construct a biometric cryptosystem using EEG signals, and evaluate it using our own dataset recorded from 42 subjects. The rest of the paper is organized as follows. In Section 2 we present the state-of-the-art overview of related work in EEG biometrics. We describe the proposed method in Section 3. We state theorems regarding the security characteristics of the cryptographic system in Section 4. We describe the application of the method on EEG dataset in Section 5. We present the experimental results and their evaluation in Section 6. Finally, the conclusions are given in Section 7.

\section{State of the Art}

Cognitive biometrics [26] uses brain signals as the source of information for user identification (authentication). User authentication is a process that ensures and confirms a user's identity in security systems. Using EEG signals for user authentication can be effective with varying degrees of accuracy. For example, Fladby [27] used power spectral features of alpha, beta low, beta high, and theta bands from just one EEG channel of 12 subjects performing eight different tasks (from simple relaxation to counting and reading) and a custom feature based distance metric for subject discrimination, achieving an EER of $21.42 \%$. Palaniappan [28] used gamma band of visually evoked potential (VEP) signals and the neural network (NN) classifier to identify 20 individuals with an average accuracy of $99.06 \%$.

Liang et al. [29] extracted the AR features from 8 EEG channels and used Support Vector Machine (SVM) to achieve an accuracy of $45.52 \%$ to $54.96 \%$ for subject separation task and an accuracy of $48.41 \%$ to $56.07 \%$ for subject identification task. Marcel and Millán [30] implemented a Gaussian mixture model (GMM) with maximum a posteriori (MAP) estimation for 9 subjects, achieving a half total error rate (HTER) of $6.6 \%$.

Hema et al. [31] adopted feed forward NN for EEG using Power Spectral Density (PSD) features from EEG beta waves and reached an average accuracy of 94.4 to $97.5 \%$ on 6 subjects. He et al. [32] used a naïve Bayes (NB) classifier with autoregressive (AR) features and achieved a HTER of $6.7 \%$ for 4 subjects.

$\mathrm{Mu}$ and $\mathrm{Hu}$ [33] used the back-propagation $\mathrm{NN}$ on data derived from 6 channels of 3 subjects and achieved an $80.7 \%$ to $86.7 \%$ accuracy. Brigham and Kumar [34] used linear SVM classifier with the AR features and achieved accuracy of $98.96 \%$ on 122 subjects tested. Hu [35] used the NN on seven EEG signal features and obtained an $80 \%$ to $100 \%$ true acceptance rate (TAR) and a 0 to $30 \%$ false acceptance rate (FAR), while using data received from only 3 subjects.

Zúquete et al. [36] demonstrated the stability of EEG biometrics using visual stimulus to measure visual evoked potentials (VEP) and a combination of one-class classifiers (OCCs), including $k$-Nearest Neighbor $(\mathrm{kNN})$ and Support Vector Data Description (SVDD). Ashby et al. [37] used linear SVM with AR and spectral characteristics of EEG signals from 14 EEG channels and achieved 2.4\% to 5.1\% false rejection rate (FRR) and $0.7 \%$ to $1.1 \%$ FAR for 5subject authentication. Shedeed [38] used the NN on features obtained by fast Fourier transform (FFT) and wavelet packet decomposition (WPD) from 4 channels, achieving a $66 \%$ to $93 \%$ correct classification rate (CCR) using data from 3 subjects.

Chuang et al. [39] recorded single-channel EEG signals when a subject performs a custom task (e.g., singing or moving finger). The authentication system analyses the similarity between such brain data and training data to authenticate subjects, reaching about 99\% accuracy. Yeom et al. [40] 
used Gaussian kernel SVM on the signal difference and time derivative features from 18 EEG channels and managed to achieve the accuracy around $86 \%$ on 10 subjects.

Dan et al. [41] used the polynomial kernel SVM based on the AR model parameters calculated on the EEG signal, recorded a single EEG channel, and obtained an accuracy of $65 \%$ to $75 \%$ on 13 subjects.

Delpozo-Banos et al. [18] used the functional connectivity patterns to represent effective features for improving EEGbased biometric systems and classification using Convolutional Neural Network (CNN) and achieved 97.5\% accuracy in eyes-closed (EC) and $96.26 \%$ in eyes-open (EO) resting state conditions states when fusing PSD information from the parietooccipital (centroparietal in EO) parts of the brain of 10 subjects.

Abo-Zahhad et al. [42] achieved more than 99\% authentication accuracy by using single-channel EEG signals from 10 and 15 subjects. Koike-Akino et al. [43] achieved 72\% accuracy for 25-subject identification from EEG using a single $800 \mathrm{~ms}$ epoch and partial least-squares (PLS) dimensionality reduction method applied before quadratic discriminant analysis (QDA) classification.

Crobe et al. [44] obtained good results in the EEG gamma $(\mathrm{EER}=0.131$ and $\mathrm{AUC}=0.943$ in $\mathrm{EO}$ condition; $\mathrm{EER}=0.130$ and $\mathrm{AUC}=0.933$ in $\mathrm{EC}$ condition $)$ and high beta $(\mathrm{EER}=0.172$ and $\mathrm{AUC}=0.905$ in $\mathrm{EO}$ condition; $\mathrm{EER}=0.173$ and $\mathrm{AUC}=$ 0.906 in EC condition) frequency bands.

Several studies presented the fusion of EEG with other modalities to get a multimodal biometric system such as in $[45,46]$. Also see a survey of security and privacy challenges in BCI applications in [47]. EEG-based authentication was also considered as a part of smart driving systems to verify the driver's identity on demand [48]. However, using EEG brainwaves for authentication might result in risks for the privacy of users. For example, authors in [49] propose an authentication system that verifies an individual EEG signal when a subject performs a custom task. They also design an attack model by impersonating the thoughts of subjects to test the robustness of the authentication system. An adversary also can attack the authentication system via synthetic EEG signals, which are generated using a model based on the historical EEG data from a subject [50].

\section{Description of EEG Biometry Method}

First, we provide definitions required for understating of the biometric authentication method as given in [25].

Definition 1 (discrete logarithm). Let $G$ be a finite cyclic group of order $n$. Let $g$ be a generator of $G$ and let $h \in G$. The discrete logarithm of $h$ to the base $g, \log _{g} h$, is the unique integer $u, 0 \leq u \leq n-1$, such that $h=g^{u}$.

Definition 2 (discrete logarithm problem (DLP)). Given a prime number $p$, a generator of $g$ of $z_{p}^{*}$, and an element $h \in z_{p}^{*}$, find the integer $u$ such that $h=g^{u}(\bmod p)$.

Definition 3 (block code). A block code $C(n, k)$ over an alphabet $A^{*}$ of $w$ symbols is a set of $w^{k} n$-vectors called codewords. Associated with the code is an encoder $\{0,1\}^{k} \rightarrow$ $C$ which maps a message $M$, a $k$-tuple, to its associated codeword.

Definition 4 (decoding function). Let $C(n, k)$ be a block code set with $w=\{0,1\}$. A decoding function $f_{d}:\{0,1\}^{n} \rightarrow C \cup$ $\varepsilon$ maps a message $c^{\prime}$, a $n$-tuple, to correct codeword $c$, if $c^{\prime}$ and $c$ are sufficiently close according to appropriate metric. Otherwise, it maps it to invalid codeword $\varepsilon$.

Definition 5 (hamming distance). Given code set $C(n, k)$, the Hamming distance between two words $c_{i}$ and $c_{j}$ from the code set $C$ is given by

$$
H\left(c_{i}, c_{j}\right)=\frac{1}{n} \sum_{r=1}^{n}\left|c_{i}^{r}-c_{j}^{r}\right| .
$$

Definition 6 (error correction threshold). Error correction threshold $t_{\sigma}$ of the error-correcting code $C(n, k)$ is the largest number of errors that can be corrected in the corrupted codeword.

Definition 7 (statistical distance). Let $X_{1}$ and $X_{2}$ be two random variables over the same space $\Psi$, and let $P_{1}$ and $P_{2}$ be their discrete probability distribution functions (PDFs). Then, the statistical distance between $P_{1}$ and $P_{2}$ is as follows:

$$
D\left(P_{1}, P_{2}\right)=\sum_{\psi \in \Psi}\left|\operatorname{Pr}\left(X_{1}=\psi\right)-\operatorname{Pr}\left(X_{2}=\psi\right)\right| .
$$

Definition 8 (Bose-Chaudhuri-Hocquenghem (BCH) codes). Let $\alpha$ be a primitive element of Galois field $\operatorname{GF}\left(q^{m}\right)$. For any positive integer $i$, let $m_{i}(x)$ be the minimal polynomial of $\alpha_{i}$ over $\operatorname{GF}\left(q^{m}\right)$. The generator polynomial of the $\mathrm{BCH}$ code is defined as the least common multiple $P(x)=$ $\operatorname{lcm}\left(m_{1}(x), \ldots, m_{d-1}(x)\right)$.

The method, proposed by [25] and adopted here for EEG biometry, consists of three procedures: (1) Setup, which outputs a public key, (2) Commit, which takes as input and the message and outputs commitment to be sent and the opening value to be used for message verification, and (3) Open, which outputs true if verification succeeds or false otherwise. Three actors participate: the sender Alice, the receiver Bob, and the trusted third party Trent, who generated system parameters and publishes it to Alice and Bob parties.

Let $M$ be the space of messages to commit to. The first stage is Setup stage (see Algorithm 1), where Trent generates and sends the keys to Alice and Bob. The second stage is Commit stage, where Alice sends Bob its commitment for a private message $m \in M$ and secretly holds an opening value. The third stage is Open stage, where Alice sends Bob the original message $m \in M$ along with the opening value, so that Bob can verify that the message committed in the first stage was indeed $m \in M$.

Definition 9 (commitment function). First we define the commitment function $F:\left(\{0,1\}^{n} \times\{0,1\}^{n}\right) \rightarrow\left(\{0,1\}^{n} \times\right.$ $\left.\{0,1\}^{n}\right)$, defined as $F(c, x)=(\varphi, \delta)$; here $\varphi=F_{k}(m, x)=$ $g^{m} h^{x}(\bmod p)$ and $\delta=x-c$ is the difference vector. 
Input: Security parameter $k$.

(1) Generate randomly two prime numbers $p$ and $q$ of length $k$, such that $p=1(\bmod q)$

(2) Choose randomly $1 \leq a \leq p-1$

(3) Compute $g=a^{(p-q) / 2} \neq 1(\bmod p)$

(4) Choose randomly $1 \leq b \leq q-1$

(5) Compute $h=g^{b} \neq 1(\bmod p)$

Output: Parameters $p, q, g, h$

Algorithm 1: Initialization.

Definition 10 (commitment protocol). Commitment proto$\operatorname{col} \pi$ is a scheme (for a message space $M$ ) defined by a triple (Setup, Commit, Open) such that

(a) $(p, q, g, h) \leftarrow \operatorname{Setup}(\cdot)$ generates the public commitment key,

(b) for any $m \in M,(\varphi, \delta) \leftarrow \operatorname{Commit}_{(p, q, g, h)}(m)$ is the commitment/opening pair for $m$,

(c) $\operatorname{Open}_{(p, q, g, h)}(\varphi, \delta) \rightarrow m \in M \cup\{\varepsilon\}$, where $\varepsilon$ is returned if $(\varphi, \delta)$ is not a valid commitment to any message.

To set the system parameters, Trent executes the following procedure.

\section{Setup Procedure}

(1) Trent generates two prime numbers $p$ and $q$ such that $p=1(\bmod q)$.

(2) Trent finds a random generator $g \in G_{q} \backslash\{1\}$, where $g \in G_{q}$ is a subgroup of the order $q$ in $Z_{p}^{*}$.

(3) Trent computes an element $h=g^{a} \in Z_{p}^{*} \backslash\{1\}$, where $a \in Z_{q}$ that is randomly chosen ( $h$ is a generator element of $\left.G_{q}\right)$.

(4) Trent sends the system parameters $(p, q, g, h)$ to Alice and Bob.

Commit Procedure. To commit to a message $m \in M_{k} \subseteq Z_{q}$ in the message space $M_{k} \subset\{0,1\}^{k}$, Alice encodes the message into a codeword $c=g(m) \in C \subseteq\{0,1\}^{n}$, chooses a random witness $x \in X_{n} \subseteq Z_{q}$ in the witness space $X_{n} \subset\{0,1\}^{n}$, and then computes the commitment $F(c, x)=\left(g^{c} h^{x}, x-c\right)=$ $(\varphi, \delta)$. The commitment is sent to Bob.

Open Procedure. To open the commitment $(\varphi, \delta)$, Alice reveals the witness $x^{\prime}$, which is in proximity to the original $x$ using some metric distance (e.g., Hamming distance $H\left(x, x^{\prime}\right) \leq$ $\left.t_{\sigma}\right)$. Using the difference vector $\delta$ the witness $x^{\prime}$ restores the codeword $f\left(c^{\prime}\right)=f\left(x^{\prime}-\delta\right)=f\left(\left(x^{\prime}-x\right)+c\right)$ and then translates $x^{\prime \prime}=\delta+f\left(c^{\prime}\right)$. Then Bob computes the commitment $\varphi^{\prime}=F_{k}\left(f\left(c^{\prime}\right), x^{\prime \prime}\right)$ and verifies $\varphi^{\prime} \stackrel{?}{=} \varphi$. In case of failure, the commitment will not open using $x^{\prime}$. Otherwise, the commitment is successfully opened and therefore the secret message is $m=m^{\prime}=g^{-1}\left(f\left(c^{\prime}\right)\right)$.

\section{Security Properties and Analysis of the Proposed Scheme}

Let $\pi=$ (Setup, Commit, Open) be a commitment scheme, and its security properties are (i) correctness, i.e., for every message the commitment generated is valid, (ii) hiding, where any attacker cannot learn information from the commitment $c$ about the message $m$ with any advantage (perfect) or with a negligible advantage, and (iii) binding, where the message $m$ is uniquely bound to $c$ (perfect) or finding another message with the same commitment has negligible probability of success. In further analysis, we assume that both the codeword $c$ and the witness $x$ are drawn randomly from the finite set $\{0,1\}^{n}$.

Definition 11 (correctness). A commitment protocol $\pi$ defined by the quadruplet $(p, q, g, h)$ is correct if, for all messages $m \in M$, Open $(p, q, g, h)\left(\operatorname{Commit}_{(p, q, g, h)}(m)\right)=m$.

The hiding property of the biometrical scheme describes the resilience of the system against adversarial attempts performed by impostor FakeBob to crack codeword $c$ or the witness $x$. We allege that impostor FakeBob knows $F$ and can access the commitment $(\varphi, \delta)$.

The binding property represents the resistance of the system against adversarial attempts by an impostor FakeBob ${ }^{*}$ to guess a codeword $c^{\prime}$ with $H\left(x, x^{\prime}\right) \leq t_{\sigma}$, such that $F_{k}(c, x)=$ $F_{k}\left(c^{\prime}, x^{\prime}\right)=\varphi$, for some $x, x^{\prime} \in X$. [51]:

For hiding and binding, we have two different adversaries

(i) the unhider $\mathrm{U}$, which plays the hiding game and has two abstract procedures, one to choose a pair of messages and another to guess which of the two messages corresponds to a given commitment;

(ii) the binder $\mathrm{B}$, which plays the binding game and has only a procedure to output two different pairs (message, opening value) that bind to the same commitment.

A commitment protocol satisfies the hiding security property if no adversary exists such that the probability of winning the hiding game is (significantly) better than a blind guess [51]. If this is true, the committer is guaranteed that no information can be inferred by the commitment itself. 


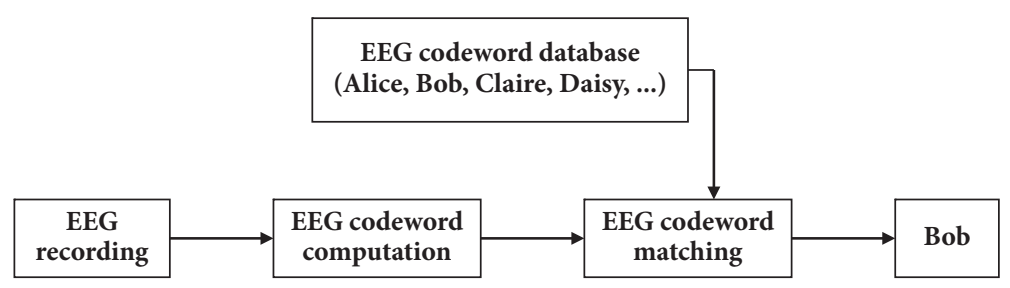

FIGURE 1: EEG-based user identification/authentication framework.

Definition 12 (hiding). Let $\pi=$ (Setup, Commit, Open) be a commitment protocol. Then we can define the hiding properties for adversary $U$ as $\operatorname{Pr}\left(G_{\pi}^{H}(n)=1\right)=1 / 2$.

Hiding Game. The hiding game $G_{\pi}^{H}$ runs as follows:

(1) The adversary $U$ is given the output of Setup procedure and asked to choose two messages.

(2) The game randomly selects one of them and calls Commit procedure to compute its commitment.

(3) The adversary $U$ is asked to guess which one of the two messages the commitment corresponds to.

(4) The game outputs 1 if the guess of the adversary $U$ is correct.

A commitment protocol satisfies the binding security property if no adversary exists such that the probability of winning the binding game is higher than negligible [51]. If this is true, the receiver is guaranteed that the value committed cannot be changed.

Definition 13 (binding). Let $\pi=$ (Setup, Commit, Open) be a commitment protocol. Then one can define the binding properties for each adversary B as $\operatorname{Pr}\left(G_{\pi}^{B}(n)=1\right)=0$.

Binding Game. The binding game $G_{\pi}^{B}$ runs as follows:

(1) The adversary $B$ is given the output of Setup procedure and asked to bind two messages to the same commitment value.

(2) The game outputs 1 if the two messages differ and the commitment is valid for both the messages, that is, if both can be verified by calling the Open procedure.

\section{Application of the Method in EEG-Based Biometric System}

Here we present the biometric cryptosystem using the EEG signals. Its implementation consists of the system initialization stage, the enrolment stage, and the authentication stage as represented in Figure 1.

At the start of enrolment (see Algorithm 3), the user EEG biometrics is acquired, and feature extraction is performed using the EEG encoding algorithm, which outputs a 400bit EEG code. We use the EEG features derived from the covariance matrix of EEG data from different EEG channels in the 10-20 international system. The covariance matrix is calculated from $N$ channels in matrix as follows:

$$
\operatorname{cov}(X)=\frac{1}{N} \sum_{k=1}^{N}\left(X_{i, k}-X_{i}\right)\left(X_{j, k}-X_{j}\right)
$$

where $X_{i}$ holds the mean of all observations in the respective EEG channels.

Next, we compute $z$-scores of the values in the covariance matrix as follows:

$$
z_{i, j}=\frac{\operatorname{cov}_{i, j}-(1 / N) \sum_{i=1}^{N} \operatorname{cov}_{i, j}}{\sqrt{\sum_{i=1}^{N}\left(\operatorname{cov}_{i, j}-(1 / N) \sum_{i=1}^{N} \operatorname{cov}_{i, j}\right)^{2} /(N-1)}} ;
$$

here $\operatorname{cov}_{i, j}$ is an element of the covariance matrix.

And perform normalization of $z$-score values of the covariance matrix into the range $[0,1]$ as follows:

$$
Z_{\text {norm }}=\frac{Z-\min (Z)}{\max (Z)-\min (Z)} .
$$

Finally, we perform the binarization of data using thresholding as follows:

$$
Z_{\text {bit }}(i, j)= \begin{cases}0, & {\left[z_{i, j}<0.5\right]} \\ 1, & {\left[z_{i, j} \geq 0.5\right]}\end{cases}
$$

here $[\cdot]$ is the Iverson bracket operator.

The result is a matrix that contains binary codeword of 400 bit length (obtained from $20 \times 20$ covariance matrix). The procedure is summarized in Algorithm 2.

At the same time, a random cryptographic key $\kappa \in\{0,1\}^{k}$ is prepared using a $\mathrm{BCH}(\ldots, k)$ error correction encoded function $\{0,1\}^{k} \rightarrow C$. The result is a codeword $c \in$ $\operatorname{BCH}(\ldots, k)$, which is combined with reference EEG code (both have 400 bits of length).

Authentication phase is described in Algorithm 4. The input EEG biometric $B_{\mathrm{EEG}}$ is acquired from a person, resulting in a test EEG code $x_{\text {test }}$. The test EEG code $x_{\text {test }}$ with "exclusive OR" denoted as $\oplus$ extracts the codeword $\widehat{c}=\left(x_{\text {test }} \oplus\right.$ $\left.x_{\text {ref }}\right) \oplus c$. Once it is extracted, the error correction decoded function of $\mathrm{BCH}(\ldots, k)$ is used to compute $f(\widehat{c})=f\left(x_{\text {test }} \otimes \delta\right)$. Function $f(\bar{c})$ is used to compute $x_{\text {test }}^{\prime}=\delta \otimes f(\bar{c})=x_{\text {ref }}(c \oplus$ $f(\widehat{c}))$. Nonvalid user will receive a codeword $f(\widehat{c})$, such that $H(f(\widehat{c}), c)>t_{\sigma}$. Then $\varphi^{\prime}=F_{k}\left(f(\widehat{c}), x_{\text {test }}^{\prime}\right)$ is computed and matched against the stored $\varphi$. If $\varphi^{\prime}=\varphi$, then the sample $x_{\text {test }}$ is accepted and the key $\kappa$ is released. Otherwise, the identity of a person is rejected.

The biometric scheme is summarized in Figure 2.

\section{Experimental Results and Discussion}

The implementation of the proposed scheme was made in MATLAB 8.6.0.267246 (R2015b) on an Intel (R) Core (TM) 
Input: EEG channel signal values

(1) Calculate covariance matrix of EEG channels.

(2) Calculate $z$-scores of covariance matrix values.

(3) Normalize $z$-scores.

(4) Binarize $z$-scores into EEG code using zero value as threshold.

Output: EEG code $x_{\text {ref }}$

Algorithm 2: Encoding.

Input: EEG biometric $B_{\mathrm{EEG}}$ and cryptographic key $\kappa$.

(1) Extract EEG code $x_{\text {ref }}$ from the EEG biometric data $B_{\mathrm{EEG}}$.

(2) Prepare the cryptographic key $\kappa$ using $\mathrm{BCH}$ codes and obtain the codeword $c$.

(3) Compute the difference vector $\delta=x_{\text {ref }} \oplus c$.

(4) Compute the commitment $\varphi=F_{k}\left(c, x_{\text {ref }}\right)$.

Output: $(\varphi, \delta)$

Algorithm 3: Enrolment.

Input: EEG biometric $B_{\mathrm{EEG}}$ and fuzzy commitment $(\varphi, \delta)$.

(1) Extract EEG code $x_{\text {test }}$ from EEG biometric $B_{\mathrm{EEG}}$.

(2) Compute the codeword $f(\bar{c})=f\left(x_{\text {test }} \otimes \delta\right)$

(3) Compute $x_{\text {test }}^{\prime}=\delta \otimes f(\bar{c})$

(4) Compute $\varphi^{\prime}=F_{k}\left(f(\widehat{c}), x_{\text {test }}^{\prime}\right)$

(5) Check $\varphi^{\prime} \stackrel{?}{=} \varphi$

Output: The user is authenticated or rejected.

Algorithm 4: Authentication.

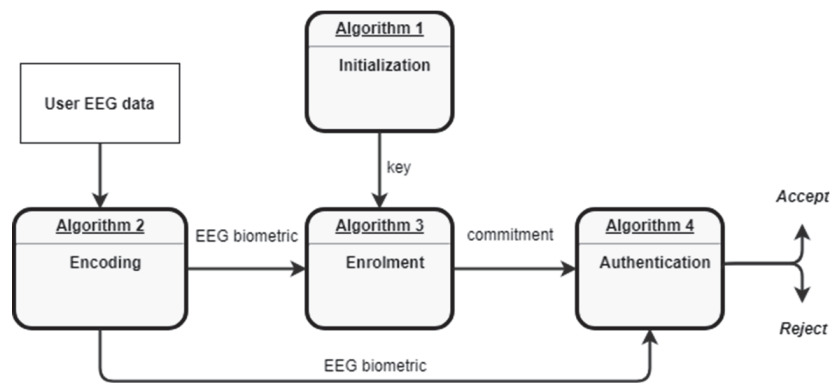

FIGURE 2: Summary of the proposed EEG biometric scheme.

i5-4590 CPU (x64), running at $3.30 \mathrm{GHz}$ with $12 \mathrm{~GB}$ of RAM in Windows 10 Enterprise ver. 1709. For the performance evaluation, we have used a dataset that consists of 65 EEG samples from 42 different subjects, where each sample consisted of 1000 signal values. The number of subjects satisfies the condition of Lazar et al. [52], who stated that studies using data collected from 20 or more participants are more convincing than those performed with a lesser number of participants. The EEG data we use in this study was collected from 42 healthy adults. During data collection, the subjects were instructed to lie still on a table and breathe normally. The data was collected using a medical-grade EEG device from

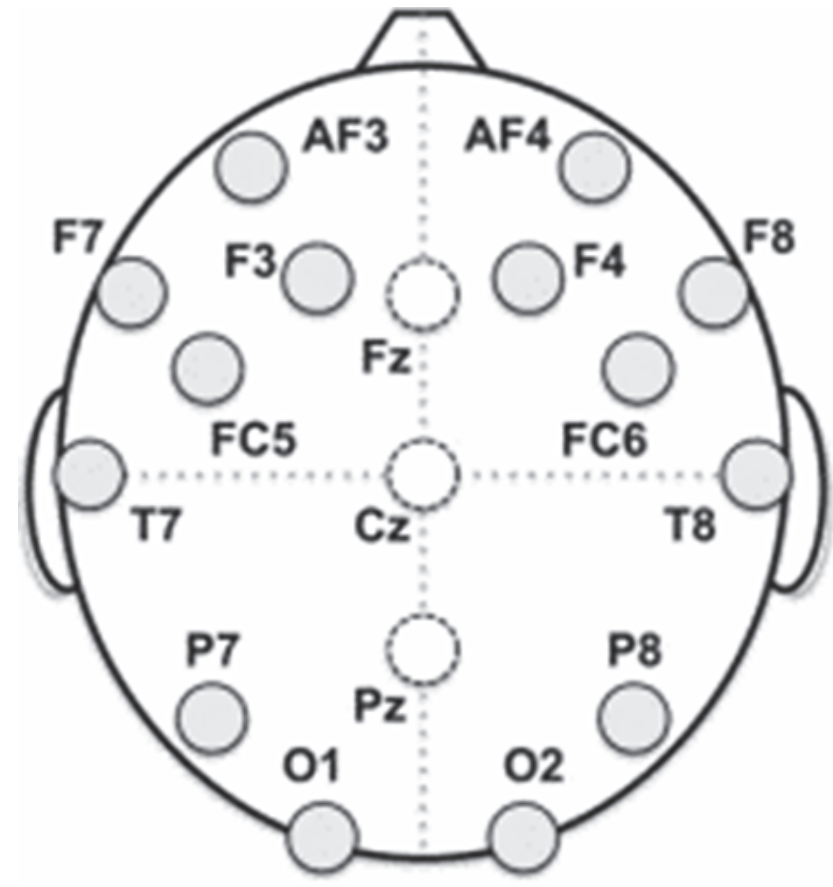

FIGURE 3: Electrode locations for collection of EEG data.

the electrodes attached to subjects following the international 10-20 standard, which are depicted as circles in Figure 3. The sampling rate was $256 \mathrm{~s}^{-1}$.

To perform code matching, we computed the Hamming distance between two EEG codewords $A$ and $B$ as follows:

$$
H=\frac{1}{n} \sum_{i=1}^{n}\left(\operatorname{code}\left(A_{i}\right) \oplus \operatorname{code}\left(B_{i}\right)\right)
$$

here $\operatorname{code}\left(A_{i}\right)$ and $\operatorname{code}\left(B_{i}\right)$ are the $i$ th bit in EEG codes of persons $A$ and $B$, respectively. 


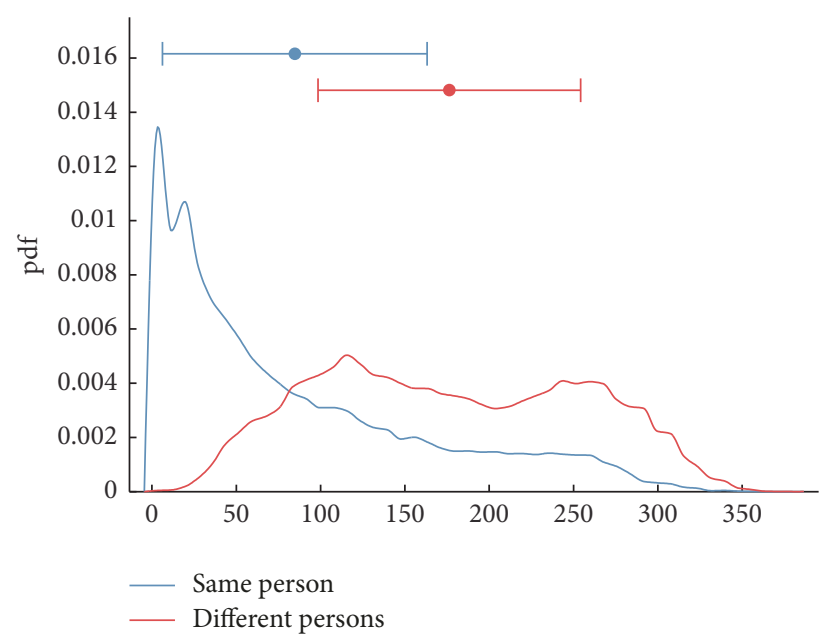

FIgURe 4: Probability density functions Hamming distances between the same person and the different persons.

The intraperson Hamming distances have been computed using EEG samples from the same subjects, while the interperson Hamming distances were computed using samples from different subjects. We carried out 65 comparisons for the same subjects and 118,335 comparisons between different subjects. The result of the probability distribution function (pdf) of the intraperson and interperson Hamming distances is shown in Figure 4. One can see that up to 87 bits of error (intersection of both graphs) are tolerated. [53].

We use the following scenarios as suggested by Gui et al.

Scenario 1 . The aim is to identify correctly each of the 42 subjects participating in the study. The training and testing datasets include data from all 42 subjects and the classification outcome belongs to one of 42 classes.

Scenario 2. The aim is to identify one subject versus all other 41 subjects. There are only two classes: positive (target subject) and negative (all other subjects). The training dataset was combined using the data from all subjects and the performing resampling so that both classes are balanced.

Evaluation. Following the suggestion of Jorgensen and $\mathrm{Yu}$ [54], we use False Accept Rate (FAR), False Reject Rate (FRR), and Equal Error Rate (EER) as key effectiveness metrics of the biometric system. FAR and FRR describe whether the system correctly identifies the subject. ERR specifies the error rate where the values of FAR and FRR become equal. The metrics are calculated as follows:

$$
\begin{aligned}
& \text { FRR }=\frac{|\mathrm{FR}|}{|\mathrm{AA}|}, \\
& \mathrm{FAR}=\frac{|\mathrm{FA}|}{|\mathrm{IA}|}
\end{aligned}
$$

here $|\mathrm{FR}|$ is the number of false rejections, that is, falsely rejecting a verification attempt of a valid subject, $|\mathrm{AA}|$ is the

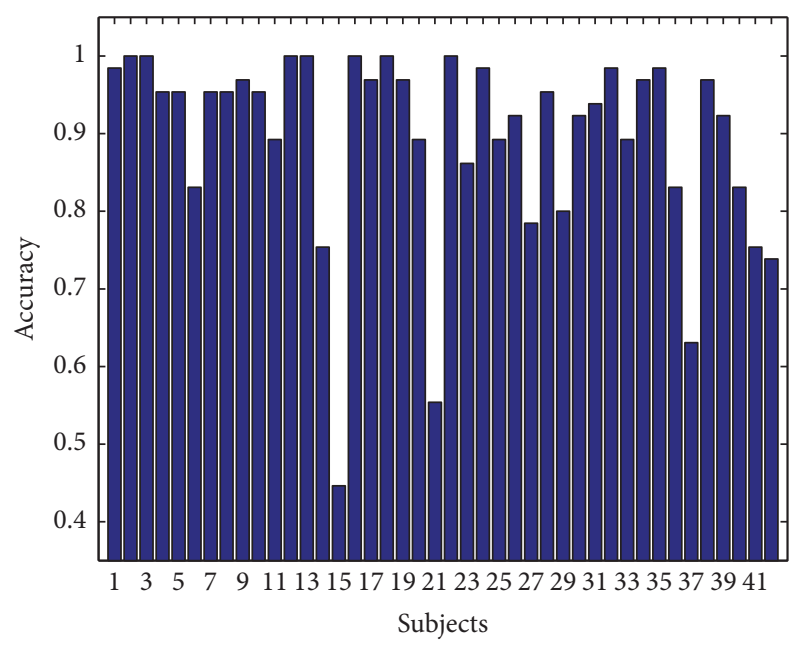

FIGURE 5: Subject-wise correct classification rate.

number of authorized attempts, $|\mathrm{FA}|$ is the number of false acceptances, i.e., falsely accepting the claim of an impostor as a valid user, and $|\mathrm{IA}|$ is the number of attempts by an impostor.

The performance is evaluated using the correct classification rate (CCR) as follows:

$$
\operatorname{CCR}=\frac{|C|}{|T|} ;
$$

here $|C|$ is the number of correct classification decisions and $|T|$ is the number of trials.

EER is defined as a unique point where FRR is equal to FAR. A lower EER indicates a more accurate system.

$$
\operatorname{EER}=\operatorname{FAR}\left(T^{*}\right)=\operatorname{FRR}\left(T^{*}\right) ;
$$

here $T^{*}=\arg \min (|\operatorname{FAR}(T)-\operatorname{FRR}(T)|)$

This ensures that the threshold found will satisfy the equality condition between FRR and FAR as closely as possible.

We have implemented both Scenarios 1 and 2 testing, as subjected by Gui et al. [53]. In Scenario 1, CCR for each of the subjects is presented in Figure 5.

Note that while the overall accuracy is quite good (mean accuracy 0.895), for some of the subjects, it was quite low (e.g., only 0.446 for subject 15$)$. This result may have been caused by the infamous BCI illiteracy effect [55]. Nevertheless, when inspecting the cumulative distribution plot of accuracy distribution (see Figure 6), we can see that $50 \%$ of subjects have accuracy higher than 0.93 , while only $10 \%$ of subjects have accuracy lower than $73 \%$.

As accuracy data is not normally distributed, the Fisher $Z$-transformation was applied to calculate population mean and standard deviation, yielding the mean accuracy of 0.892 with standard deviation of 0.135 .

The subject-wise confusion matrix is presented in Figures 7 and 8 . As the number of subjects is too high for meaningful visualization, the confusion matrix was sorted according to its diagonal value (correct hits), and the values for only 10 


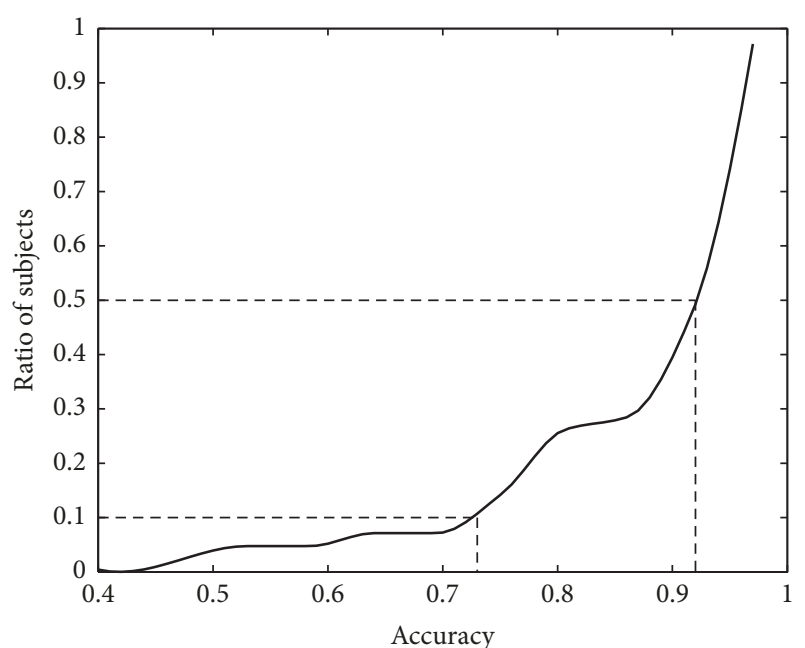

FIGURE 6: Cumulative distribution plot of accuracy distribution in subject classification.

\begin{tabular}{|c|c|c|c|c|c|c|c|c|c|c|}
\hline 15 & -0.45 & 0 & 0.05 & 0.03 & 0.03 & 0.02 & 0 & 0.02 & 0.32 & 0 \\
\hline 21 & -0.02 & 0.55 & 0.03 & 0.03 & 0.03 & 0.02 & 0 & 0 & 0 & 0 \\
\hline 37 & -0.03 & 0.02 & 0.63 & 0.02 & 0.02 & 0.02 & 0.15 & 0.02 & 0 & 0.02 \\
\hline 4 & -0.03 & 0.02 & 0 & 0.74 & 0.02 & 0.05 & 0.02 & 0.02 & 0 & 0 \\
\hline 1 & -0.03 & 0 & 0 & 0 & 0.75 & 0 & 0.02 & 0.03 & 0 & 0.03 \\
\hline 4 & -0.02 & 0 & 0 & 0.03 & 0.02 & 0.75 & 0 & 0.03 & 0 & 0 \\
\hline 2 & 0 & 0.02 & 0.06 & 0.02 & 0.02 & 0 & 0.78 & 0 & 0 & 0 \\
\hline 2 & 0 & 0 & 0 & 0 & 0.02 & 0.06 & 0 & 0.8 & 0 & 0.03 \\
\hline ? & -0.11 & 0.02 & 0 & 0 & 0 & 0 & 0 & 0 & 0.83 & 0 \\
\hline & -0.02 & 0 & 0 & 0.02 & 0.02 & 0.02 & 0 & 0 & 0 & 0.83 \\
\hline & 15 & 21 & 37 & 42 & 14 & 41 & 27 & 29 & 6 & 36 \\
\hline
\end{tabular}

FIGURE 7: Subject-wise confusion matrix of classification results in Scenario 1: 10 worst performing subjects.

\begin{tabular}{|c|c|c|c|c|c|c|c|c|c|c|c|}
\hline & 1 & 0 & 0 & 0 & 0 & 0 & 0 & 0 & 0 & 0 & 0 \\
\hline 18 & 0 & 1 & 0 & 0 & 0 & 0 & 0 & 0 & 0 & 0 & 0 \\
\hline 16 & 0 & 0 & 1 & 0 & 0 & 0 & 0 & 0 & 0 & 0 & 0 \\
\hline 1. & 0 & 0 & 0 & 1 & 0 & 0 & 0 & 0 & 0 & 0 & 0 \\
\hline 1 & 0 & 0 & 0 & 0 & 1 & 0 & 0 & 0 & 0 & 0 & 0 \\
\hline 3 & 0 & 0 & 0 & 0 & 0 & 1 & 0 & 0 & 0 & 0 & 0 \\
\hline 2 & 0 & 0 & 0 & 0 & 0 & 0 & 1 & 0 & 0 & 0 & 0 \\
\hline 3 & 0 & 0 & 0 & 0 & 0 & 0 & 0 & 0.98 & 0 & 0 & 0 \\
\hline 32 & 0 & 0 & 0 & 0 & 0 & 0 & 0 & 0 & 0.98 & 0 & 0 \\
\hline 2 & 0 & 0 & 0 & 0 & 0 & 0 & 0 & 0 & 0 & 0.98 & 0 \\
\hline & 0 & 0 & 0 & 0 & 0 & 0 & 0 & 0 & 0 & 0 & 0.98 \\
\hline
\end{tabular}

FIGURE 8: Subject-wise confusion matrix of classification results in Scenario 1: 10 best performing subjects.

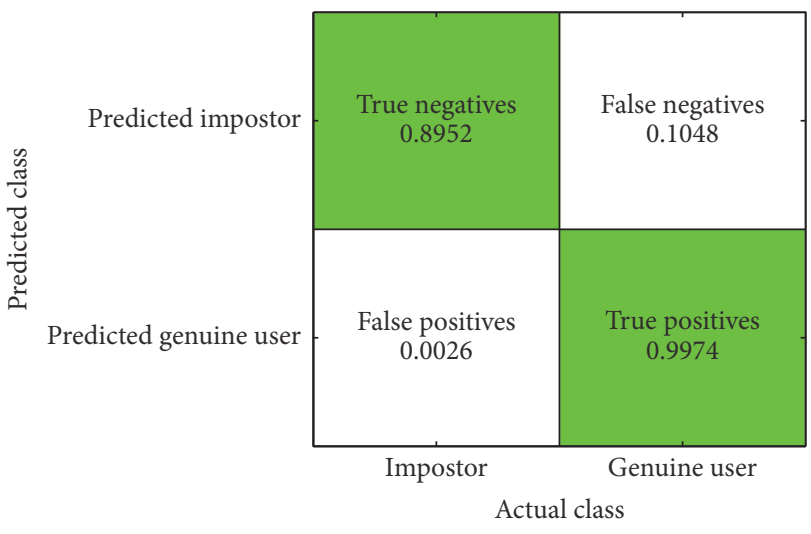

FIGURE 9: Confusion matrix of classification results in Scenario 2.

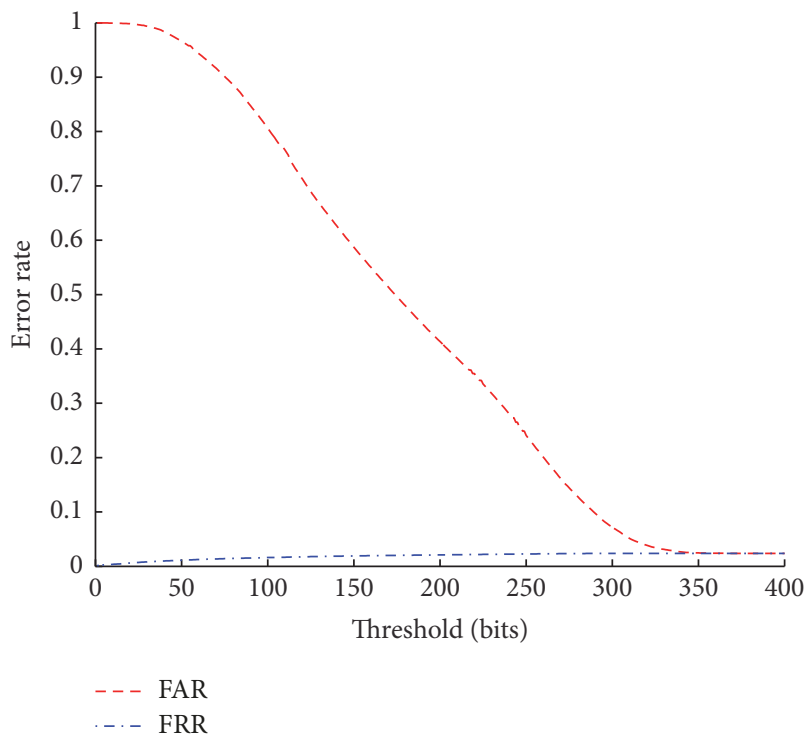

FIGURE 10: FAR and FRR of the proposed EEG biometric system.

worst performing subjects (Figure 7) and 10 best performing subjects (Figure 8 ) are shown.

For Scenario 2, the confusion matrix is presented in Figure 9. We can see that True Positive Rate (TPR) is 0.9974. We have evaluated the confusion matrix statistically using the McNemar test. Critical value at $95 \%$ significance level is 3.8415. McNemar chi-square with Yates correction is 0.001, while $p=0.966$. Therefore, the results are significant at alpha $=0.05$ level.

The values for FAR, FRR, and ERR are represented in Figure 10.

The Area Under Curve (AUC) is calculated as the area under the Receiver Operating Characteristic (ROC) [56] curve and represents discrimination, that is, the ability of the classifier to discriminate between a positive example and a negative example.

We have achieved the following results, which are summarized in Table 1. 
TABLE 1: Summary of classification results.

\begin{tabular}{lcccc}
\hline TAR & FRR & ERR & AUC & TPR \\
\hline 0.8952 & 0.026 & 0.024 & 0.9271 & 0.9974 \\
\hline
\end{tabular}

TABLE 2: Comparison of the proposed method with the Fladby's method [27].

\begin{tabular}{lcc}
\hline $\begin{array}{l}\text { EER (proposed } \\
\text { method + our } \\
\text { dataset) }\end{array}$ & $\begin{array}{c}\text { EER (Fladby method } \\
\text { + Fladby dataset) }\end{array}$ & $\begin{array}{c}\text { EER (Fladby method } \\
\text { + our dataset) }\end{array}$ \\
\hline & & 0.3059 (mean, all \\
& & channels) \\
& 0.2142 & 0.2945 (Fp1) \\
& 0.2283 (best, P4) \\
\hline
\end{tabular}

Comparison. In Table 2 and Figure 11, we compare our results with those of Fladby [27]. Note that Fladby used a simple EEG reading device (Neurosky ThinkGear) with only one channel of EEG data (Fpl), which may be affected by eye artefacts. Sampling frequency was only $128 \mathrm{~Hz}$, and 20 seconds of signal samples for each of eight different tasks was used for authentication, which is unpractical for many applications. Nevertheless, the method of Fladby [27], which employs widely used power spectral features of EEG bands, can be considered as a baseline, against which our method could be compared. We have thoroughly replicated the conditions of the experiment by Fladby on our dataset, using the same number of samples (2560) for each snippet of subject EGG data and a feature based distance metric to discriminate between genuine and fraudulent authentication results, and calculated the EER value. Note that our method uses all 20 EEG channels of the 10-20 international system, while Fladby used only one EEG channel. Nevertheless, we have replicated the calculations of the Fladby's method on each EEG channel to make a fair comparison. The results are presented in Table 2 as well as in Figure 11. Fladby's method achieved mean ERR of 0.3059 , while the Fpl channel originally used by Fladby achieved an ERR of 0.2945 , and best ERR was achieved using the P4 channel (0.2283). Note that we could not apply our method on Fladby's data, because it is not available.

Based on the presented comparison, we can claim that the proposed method achieved better results for subject authentication than the Fladby [27] method.

\section{Conclusion}

This paper presents a secure cryptographic authentication scheme for EEG-based biometrics based on the fuzzy commitment scheme and the error-correcting Bose-ChaudhuriHocquenghem $(\mathrm{BCH})$ codes. The EEG features are derived from the covariance matrix of EEG data from different EEG channels in the 10-20 international system. The biometric system was evaluated using the EEG dataset obtained from 42 subjects. The experimental results show that the system can generate up to 400 bits of cryptographic key from the EEG codes, while tolerating up to 87 bits of error. The performance of the biometric cryptosystem is an Equal Error Rate (EER)

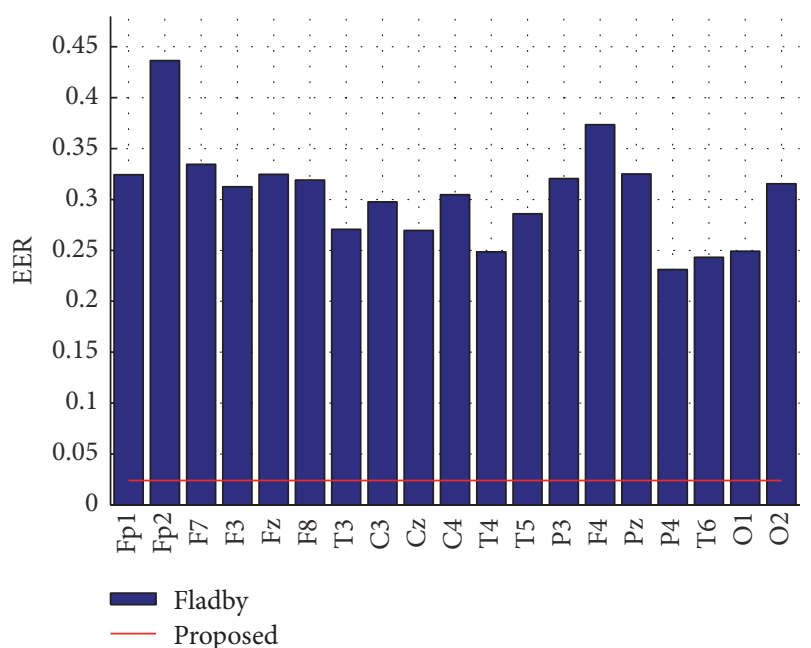

FIGURE 11: Comparison of EER of our method and Fladby's method [27] for each EEG channel.

of 0.024, True Positive Rate (TPR) of 0.9974, and Area Under Curve (AUC) of 0.927.

\section{Conflicts of Interest}

The authors declare that there are no conflicts of interest regarding the publication of this paper.

\section{Acknowledgments}

The authors would like to acknowledge the support from the Rector pro-quality Grant no. 09/010/RGJ18/0034 at the Silesian University of Technology. The authors would also like to thank professor A. Vainoras of Lithuanian University of Health Sciences for kindly provided EEG dataset.

\section{References}

[1] T. M. Vaughan, W. J. Heetderks, L. J. Trejo et al., Brain-Computer Interface Technology: A Review of The Second International Meeting, 2003.

[2] C. G. Lim, T. S. Lee, C. Guan et al., "A brain-computer interface based attention training program for treating attention deficit hyperactivity disorder," PLOS ONE, vol. 7, no. 10, Article ID e46692, 2012.

[3] M. Ahn, M. Lee, J. Choi, and S. C. Jun, "A review of braincomputer interface games and an opinion survey from researchers, developers and users," Sensors, vol. 14, no. 8, pp. 14601-14633, 2014.

[4] I. Martišius and R. Damaševičius, "A prototype SSVEP based real time BCI gaming system," Computational Intelligence \& Neuroscience, vol. 2016, Article ID 3861425, 15 pages, 2016.

[5] Y. Iidal, D. Tsutsumi, S. Saeki, Y. Ootsuka, T. Hashimoto, and R. Horie, "The effect of immersive head mounted display on a brain computer interface game," Advances in Intelligent Systems and Computing, vol. 483, pp. 211-219, 2017.

[6] J. D. Woodward, N. M. Orlans, and P. T. Higgins, Biometrics, McGraw-Hill, Berkeley, Calif, USA, 1953. 
[7] A. S. Danko and G. C. Fernández, "My brain is my passport. Verify me," in Proceedings of the 2016 IEEE International Conference on Consumer Electronics (ICCE), pp. 19-22, Las Vegas, NV, USA, 2002.

[8] C.-C. Hsu and P.-T. Shih, "A novel sleep apnea detection system in electroencephalogram using frequency variation," Expert Systems with Applications, vol. 38, no. 5, pp. 6014-6024, 2011.

[9] M. Taghavi, R. Boostani, M. Sabeti, and S. M. A. Taghavi, "Usefulness of approximate entropy in the diagnosis of schizophrenia," Iranian Journal of Psychiatry and Behavioral Sciences, vol. 5, no. 2, pp. 62-70, 2011.

[10] M. Li, W. Chen, and T. Zhang, "Classification of epilepsy EEG signals using DWT-based envelope analysis and neural network ensemble," Biomedical Signal Processing and Control, vol. 31, pp. 357-365, 2017.

[11] U. A. Qidwai and M. Shakir, "Fuzzy classification-based control of wheelchair using EEG data to assist people with disabilities," in Proceedings of the 19th International Conference on Neural Information Processing, ICONIP 2012, vol. 7666 of Springer Lecture Notes in Computer Science 7666, pp. 458-467, Doha, Qatar, 2012.

[12] R. N. Khushaba, C. Wise, S. Kodagoda, J. Louviere, B. E. Kahn, and C. Townsend, "Consumer neuroscience: Assessing the brain response to marketing stimuli using electroencephalogram (EEG) and eye tracking," Expert Systems with Applications, vol. 40, no. 9, pp. 3803-3812, 2013.

[13] Researchbeam, "Global EEG and ECG Biometrics Market 20162020," 2016.

[14] S. Z. Li and A. K. Jain, "EEG biometrics," Encyclopedia of Biometrics, pp. 389-396, 2015.

[15] H. Van Dis, M. Corner, R. Dapper, G. Hanewald, and H. Kok, "Individual differences in the human electroencephalogram during quiet wakefulness," Electroencephalography and Clinical Neurophysiology, vol. 47, no. 1, pp. 87-94, 1979.

[16] C. E. M. Van Beijsterveldt and G. C. M. Van Baal, “Twin and family studies of the human electroencephalogram: A review and a meta-analysis," Biological Psychology, vol. 61, no. 1-2, pp. $111-138,2002$

[17] E. Maiorana, D. La Rocca, and P. Campisi, "On the permanence of EEG signals for biometric recognition," IEEE Transactions on Information Forensics and Security, vol. 11, no. 1, pp. 163-175, 2016.

[18] M. Delpozo-Banos, C. M. Travieso, C. T. Weidemann, and J. B. Alonso, "EEG biometric identification: A thorough exploration of the time-frequency domain," Journal of Neural Engineering, vol. 12, no. 5, Article ID 056019, 2015.

[19] D. L. Rocca, P. Campisi, B. Vegso et al., "Human brain distinctiveness based on EEG spectral coherence connectivity," IEEE Transactions on Biomedical Engineering, vol. 61, no. 9, pp. 24062412, 2014.

[20] M. Fraschini, A. Hillebrand, M. Demuru, L. Didaci, and G. L. Marcialis, "An EEG-based biometric system using eigenvector centrality in resting state brain networks," IEEE Signal Processing Letters, vol. 22, no. 6, pp. 666-670, 2015.

[21] A. Juels and M. Wattenberg, "A fuzzy commitment scheme," in Proceedings of the 1999 6th ACM Conference on Computer and Communications Security (ACM CCS), pp. 28-36, November 1999.

[22] A. Juels and M. Sudan, "A fuzzy vault scheme," Designs, Codes and Cryptography. An International Journal, vol. 38, no. 2, pp. 237-257, 2006.
[23] F. Hao, R. Anderson, and J. Daugman, "Combining crypto with biometrics effectively," IEEE Transactions on Computers, vol. 55, no. 9, pp. 1081-1088, 2006.

[24] C. Rathgeb and A. Uhl, "A survey on biometric cryptosystems and cancelable biometrics," EURASIP Journal on Information Security, vol. 2011, article no. 3, pp. 1-25, 2011.

[25] A. A. Al-Saggaf, "Secure method for combining cryptography with iris biometrics," Journal of Universal Computer Science, 2018, Special Issue on Advances in Security and Privacy of Multimodal Interfaces.

[26] K. Revett, F. Deravi, and K. Sirlantzis, "Biosignals for user authentication - towards cognitive biometrics?" in Proceedings of the 2010 International Conference on Emerging Security Technologies, EST 2010, Robots and Security, ROBOSEC 2010, Learning and Adaptive Behavior in Robotic Systems, LAB-RS 2010, pp. 71-76, Canterbury, UK, September 2010.

[27] K. Fladby, Brain Wave Based Authentication, Gjøvik University College, 2008.

[28] R. Palaniappan, "Method of identifying individuals using VEP signals and neural network," IEE Proceedings Science, Measurement and Technology, vol. 151, no. 1, pp. 16-20, 2004.

[29] N. Liang, P. Saratchandran, G. Huang, and N. Sundararajan, "Classification of mental tasks from EEG signals using extreme learning machine," International Journal of Neural Systems, vol. 16, no. 1, pp. 29-38, 2006.

[30] S. Marcel and J. D. R. Millán, "Person authentication using brainwaves (EEG) and maximum a posteriori model adaptation," IEEE Transactions on Pattern Analysis and Machine Intelligence, vol. 29, no. 4, pp. 743-752, 2007.

[31] C. R. Hema, M. P. Paulraj, and H. Kaur, "Brain signatures: a modality for biometric authentication," in Proceedings of the International Conference on Electronic Design (ICED '08), pp. 13, Penang, Malaysia, December 2008.

[32] C. He, X. Lv, and Z. J. Wang, "Hashing the mAR coefficients from EEG data for person authentication," in Proceedings of the IEEE International Conference on Acoustics, Speech, and Signal Processing (ICASSP '09), pp. 1445-1448, Taipei, Taiwan, April 2009.

[33] Z. Mu and J. Hu, "Research of EEG identification computing based on AR model," in Proceedings of the 2009 International Conference on Future BioMedical Information Engineering, FBIE 2009, pp. 366-368, Sanya, China, December 2009.

[34] K. Brigham and B. V. K. V. Kumar, "Subject identification from Electroencephalogram (EEG) signals during imagined speech," in Proceedings of the 4th IEEE International Conference on Biometrics: Theory, Applications and Systems, BTAS 2010, pp. 18, September 2010.

[35] J.-F. Hu, "Biometric system based on EEG signals by feature combination," in Proceedings of the 2010 International Conference on Measuring Technology and Mechatronics Automation (ICMTMA 2010), pp. 752-755, Changsha City, China, March 2010.

[36] A. Zúquete, B. Quintela, J. Cunha, and A. Zúquete, "Biometric authentication with electroencephalograms: evaluation of its suitability using visual evoked potentials," in Biomedical Engineering Systems and Technologies, A. Fred, J. Filipe, and H. Gamboa, Eds., vol. 127, pp. 290-306, Springer, Heidelberg, Berlin, Germany, 2011.

[37] C. Ashby, A. Bhatia, F. Tenore, and J. Vogelstein, "Low-cost electroencephalogram (EEG) based authentication," in Proceedings of the 2011 5th International IEEE/EMBS Conference on Neural Engineering, NER 2011, pp. 442-445, Cancun, Mexico, May 2011. 
[38] H. A. Shedeed, "A new method for person identification in a biometric security system based on brain EEG signal processing," in Proceedings of the 2011 World Congress on Information and Communication Technologies, WICT 2011, pp. 1205-1210, Mumbai, India, December 2011.

[39] J. Chuang, H. Nguyen, C. Wang, and B. Johnson, "I think therefore I am: Usability and security of authentication using brainwaves," in Financial Cryptography and Data Security, vol. 7862 of Lecture Notes in Computer Science, pp. 1-16, Springer, Heidelberg, Berlin, Germany, 2013.

[40] S.-K. Yeom, H.-I. Suk, and S.-W. Lee, "Person authentication from neural activity of face-specific visual self-representation," Pattern Recognition, vol. 46, no. 4, pp. 1159-1169, 2013.

[41] Z. Dan, Z. Xifeng, and G. Qiangang, "An Identification System Based on Portable EEG Acquisition Equipment," in Proceedings of the 2013 Third International Conference on Intelligent System Design and Engineering Applications (ISDEA), pp. 281-284, Hong Kong, China, January 2013.

[42] M. Abo-Zahhad, S. M. Ahmed, and S. N. Abbas, "A new EEG acquisition protocol for biometric identification using eye blinking signals," Intelligent Systems and Applications, vol. 7, no. 6, pp. 48-54, 2015.

[43] T. Koike-Akino, R. Mahajan, T. K. Marks et al., "High-accuracy user identification using EEG biometrics," in Proceedings of the 38th Annual International Conference of the IEEE Engineering in Medicine and Biology Society, EMBC 2016, pp. 854-858, USA, August 2016.

[44] A. Crobe, M. Demuru, L. Didaci, G. L. Marcialis, and M. Fraschini, "Minimum spanning tree and k-core decomposition as measure of subject-specific EEG traits," Biomedical Physics and Engineering Express, vol. 2, no. 1, 2016.

[45] S. Barra, A. Casanova, M. Fraschini, and M. Nappi, "Fusion of physiological measures for multimodal biometric systems," Multimedia Tools and Applications, vol. 76, no. 4, pp. 4835-4847, 2017.

[46] M. Garau, M. Fraschini, L. Didaci, and G. L. Marcialis, "Experimental results on multi-modal fusion of EEG-based personal verification algorithms," in Proceedings of the 9th IAPR International Conference on Biometrics, ICB 2016, pp. 1-6, Halmstad, Sweden, June 2016.

[47] Q. Q. Li, D. Ding, and M. Conti, "Brain-Computer Interface applications: Security and privacy challenges," in Proceedings of the 2015 IEEE Conference on Communications and Network Security (CNS), pp. 663-666, Florence, Italy, September 2015.

[48] I. Nakanishi, K. Ozaki, and S. Li, "Evaluation of the brain wave as biometrics in a simulated driving environment," in Proceedings of the 2012 International Conference of the Biometrics Special Interest Group, BIOSIG 2012, pp. 1-5, Darmstadt, Germany, September 2012.

[49] B. Johnson, T. Maillart, and J. Chuang, "My thoughts are not your thoughts," in Proceedings of the 2014 ACM International Joint Conference on Pervasive and Ubiquitous Computing, UbiComp 2014, pp. 1329-1338, USA, September 2014.

[50] S. T. Archer and B. D. Pless, Stimulation Signal Generator for an Implantable Device, US Patent 6690974, 2004.

[51] R. Metere and C. Dong, "Automated cryptographic analysis of the pedersen commitment scheme," in Proceedings of the International Conference on Mathematical Methods, Models, and Architectures for Computer Network Security, MMM-ACNS 2017, vol. 10446 of Lecture Notes in Computer Science 10446, pp. 275-287, Springer, Warsaw, Poland, 2017.
[52] J. Lazar, J. H. Feng, and H. Hochheiser, Research Methods in Human-Computer Interaction, Wiley, New York, NY, USA, 2010.

[53] Q. Gui, Z. Jin, and W. Xu, "Exploring EEG-based biometrics for user identification and authentication," in Proceedings of the 2014 IEEE Signal Processing in Medicine and Biology Symposium (SPMB), pp. 1-6, Philadelphia, PA, USA, December 2014.

[54] Z. Jorgensen and T. Yu, "On mouse dynamics as a behavioral biometric for authentication," in Proceedings of the 6th International Symposium on Information, Computer and Communications Security, ASIACCS 2011, pp. 476-482, China, March 2011.

[55] C. Vidaurre and B. Blankertz, "Towards a cure for BCI illiteracy," Brain Topography, vol. 23, no. 2, pp. 194-198, 2010.

[56] H. V. Poor, An Introduction to Signal Detection and Estimation, chapter 4, Springer, New York, NY, USA, 1985. 


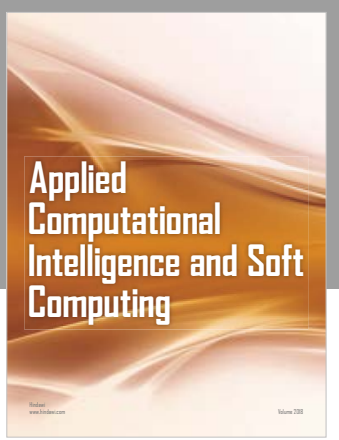

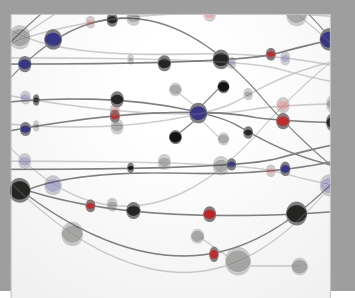

The Scientific World Journal
Submit your manuscripts at

Computing
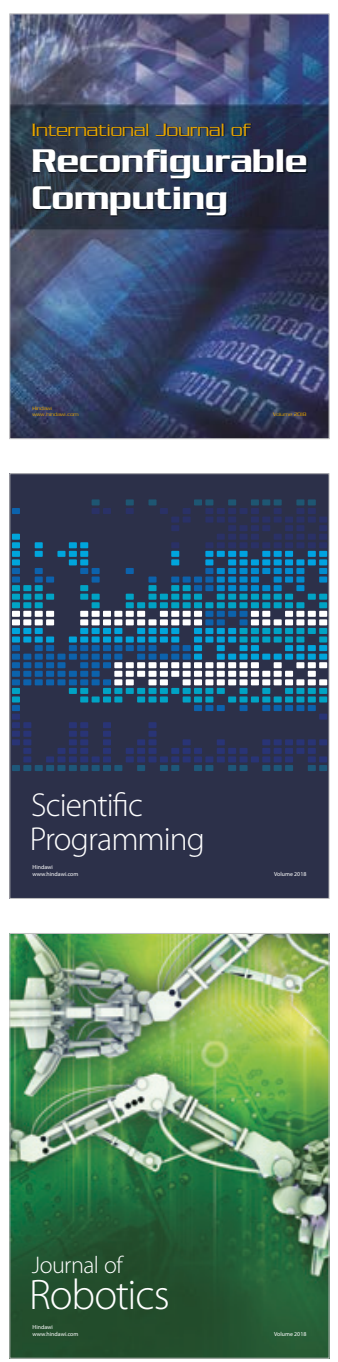

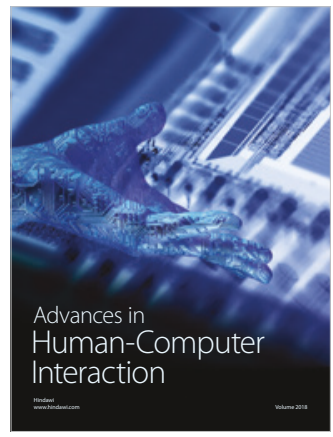

Human-Compute

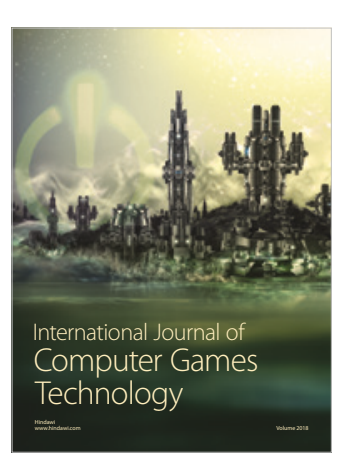

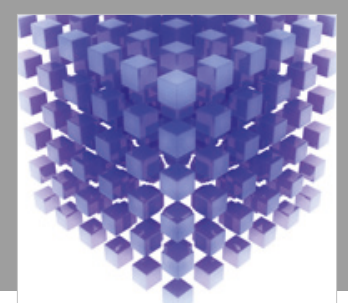

Mathematical Problems in Engineering

\section{Engincering}
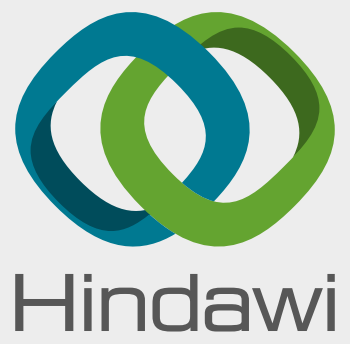

www.hindawi.com
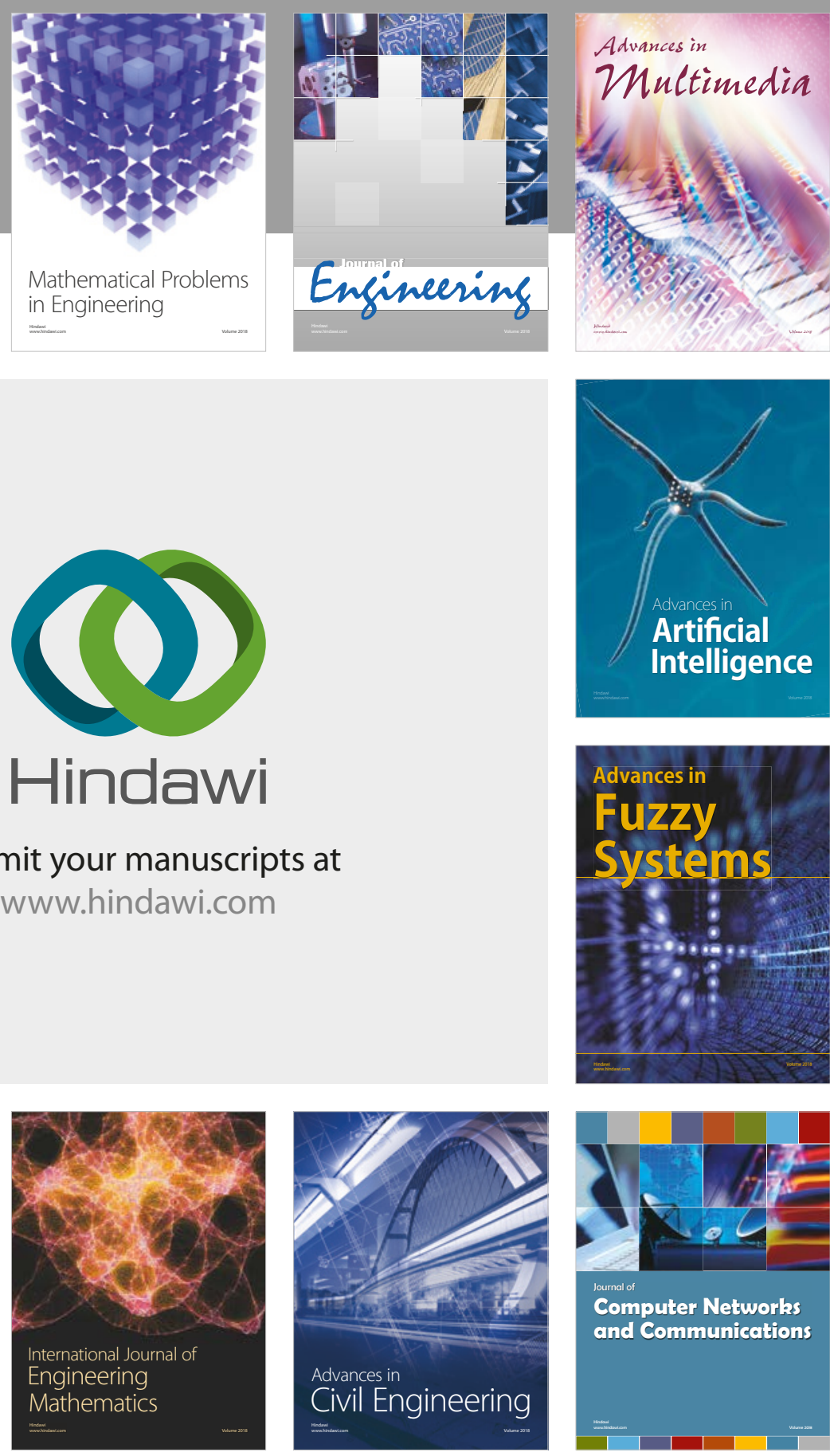

Computer Networks and Communications

Multimedia
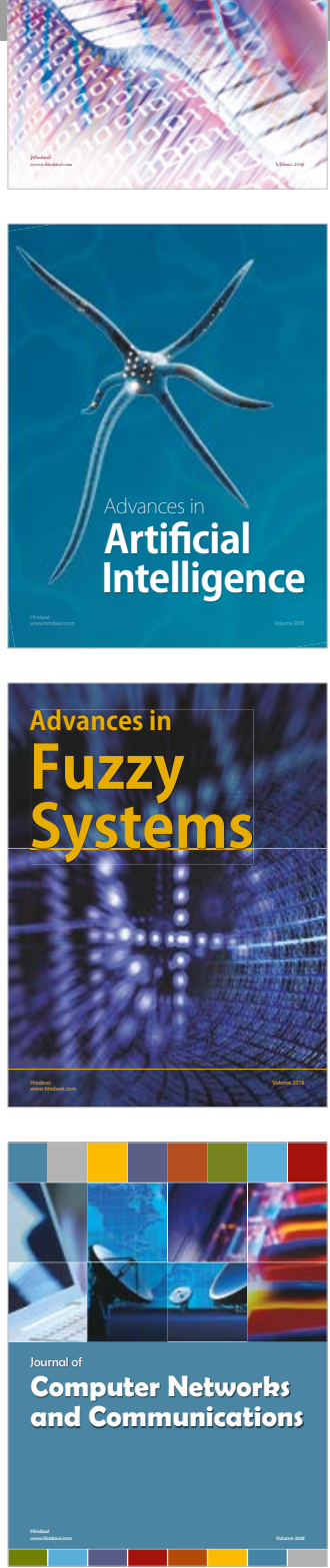

Advances in

Modelling \&

Simulation

in Engineering

interaction

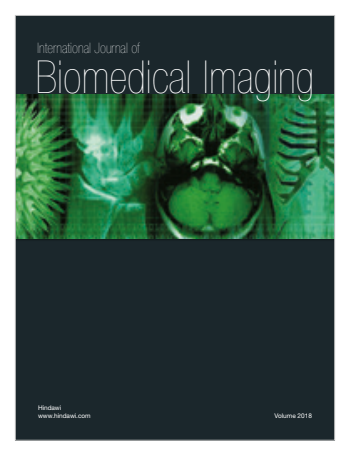

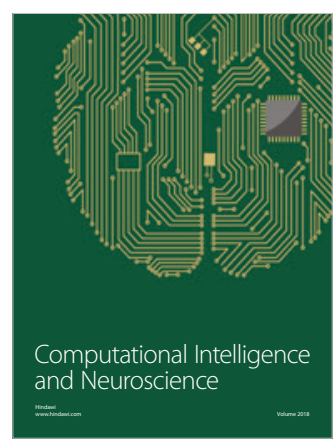

